\title{
Adaptability variations and differences in photoprotection of young leaves of two tree species of subtropical forest to the light environment
}

\author{
Z.-C. YU ${ }^{\dagger}$ (iD), Y.-N. LUO ${ }^{\dagger}$, W.-Y. SHI, W. LIN, and C.-L. PENG ${ }^{+}$ \\ Guangzhou Key Laboratory of Subtropical Biodiversity and Biomonitoring, Guangdong Provincial Key \\ Laboratory of Biotechnology for Plant Development, School of Life Sciences, South China Normal University, \\ 510631 Guangzhou, China
}

\begin{abstract}
In the high-light environment, young leaves accumulate anthocyanins as a photoprotection strategy. However, anthocyanin biosynthesis-related enzymes gene sequence is still unknown in the leaves of subtropical forest plants. There are thus few reports on the relationship between the expression level of these genes and photoprotection. In this study, Machilus chinensis and Castanopsis chinensis were taken as plant material in a subtropical forest. Non-full-length nucleotide sequences of chalcone synthase, dihydroflavonol 4-reductase, anthocyanin synthase, and $\beta$-tubulin gene were obtained by homologous and electronic cloning. The expression of those genes was verified and analyzed, and some physiological indicators were measured. The anthocyanin content and anthocyanin synthesisrelated gene expression in the young leaves of $C$. chinensis were significantly higher than that of $M$. chinensis. Although young leaves of $M$. chinensis did not accumulate anthocyanins, they showed higher antioxidants and nonphotochemical quenching (NPQ). This study indicates that anthocyanins, antioxidants, and NPQ together mediate the positive effects on photoadaptation in young leaves.
\end{abstract}

Keywords: anthocyanins; antioxidants; light environment; nonphotochemical quenching; photoprotection.

\section{Introduction}

The alternation of young leaves and mature leaves is the basis of maintaining the continuous growth of the population. So the normal growth and development of the young leaf are particularly important. However, young leaves do not always grow in a suitable environment, but usually face various stresses. These include strong light,
UV-B radiation, extreme temperature, drought, lack of nutrients, etc. (Chalker-Scott 1999). In the subtropical forest, high temperature and high light in summer are some of the main stress factors faced by plants (Faria et al. 1998, Szymańska et al. 2017). In summer, the maximum sunlight radiation in the subtropical forest canopy can reach 2,000 $\mu \mathrm{mol}\left(\right.$ photon) $\mathrm{m}^{-2} \mathrm{~s}^{-1}$, and the average temperature exceeds $36^{\circ} \mathrm{C}$, sometimes reaching $40^{\circ} \mathrm{C}$ (Zhang et al. 2016 , Yu

\section{Highlights}

- Anthocyanin biosynthesis-related gene sequence was obtained in subtropical forest plants

- The CHS, DFR, and ANS sequences were obtained by homologous and electronic cloning

- Anthocyanins, antioxidants, and NPQ together mediate photoprotection of young leaves
Received 29 August 2020

Accepted 11 January 2021

Published online 15 February 2021

${ }^{+}$Corresponding author

e-mail: pengchl@scib.ac.cn

Abbreviations: ANS - anthocyanin synthase; CHS - chalcone synthase; DFR - dihydroflavonol 4-reductase; DPPH - 1,1-diphenyl2-picrylhydrazyl; $\mathrm{F}_{\mathrm{m}}$ - maximal fluorescence yield of the dark-adapted state; $\mathrm{F}_{\mathrm{m}}$ ' - maximum fluorescence of the light-adapted state; $\mathrm{F}_{\mathrm{v}} / \mathrm{F}_{\mathrm{m}}$ - maximal quantum yield of PSII photochemistry; NPQ - nonphotochemical quenching; ROS - reactive oxygen species; TUB $\beta$-tubulin.

Acknowledgements: This work was funded by the National Natural Science Foundation of China (31870374, 31570398). The study was also supported by the Guangdong Province Natural Science Foundation (2017A030313167, 2015A030311023).

Conflict of interest: The authors declare that they have no conflict of interest.

${ }^{\dagger}$ These authors contributed equally to this work. 
et al. 2020). Due to the incomplete development of the photosynthetic apparatus in young leaves of plants, the imbalance of light energy absorption and utilization in a high-light environment is easy to reach; the excess of light energy can cause photoinhibition or photodamage (Krause et al. 1995, Murata et al. 2012). In this unfavorable environment, if no strong photoprotection strategy exists, the young leaves of plants cannot grow normally. In order to ensure the normal development of young leaves, plants have formed a complete set of defense strategies in the long-term evolution process.

Some researchers have found that about one-third of young leaves of plants in tropical forests turn red before they mature (Dominy et al. 2002), and about four-fifths of the young leaves of forest plants turn red because anthocyanin accumulated (Kong et al. 2003). The selective pressure driving anthocyanin evolution in plant leaves is still unknown. However, plant physiologists have made significant progress in elaborating the effect of anthocyanins on plant functions. Anthocyanins in plant leaves usually occur as vacuolar solutions in epidermal or mesophyll cells (Kunz et al. 1994, Gould et al. 2000, Lee and Collins 2001, Hooijmaijers and Gould 2007). However, regardless of their position in the cell, the genes of anthocyanin synthesis-related enzymes in many leaves are usually upregulated in response to one or more environmental stresses. Chalcone synthase (CHS) is the first key enzyme in anthocyanin synthesis (Deng et al. 2014), and it plays a decisive role in the formation of plant flower color and leaf color. Dihydroflavonol 4-reductase (DFR) is a key enzyme in anthocyanin synthesis, which is responsible for the reduction of flavonol to colorless anthocyanins (Nakatsuka et al. 2005a). Finally, under the catalysis of anthocyanin synthase (ANS), a variety of colorless anthocyanins are transformed into colored anthocyanins (Nakatsuka et al. 2005b). Therefore, these genes should be upregulated in the leaves of plants to reflect the impact of stress. In recent years, more attention has been paid to the photoprotective effect of leaf anthocyanins. High-light stress can induce a lot of free radicals in plant cells (Takahashi and Murata 2008, Murata et al. 2012). Anthocyanins can reduce the oxidative load of leaves in two ways. First, anthocyanins can shield part of the high energy quantum light from entering the photosynthetic system and prevent or slow down the photooxidation damage caused by chlorophyll bleaching (Kato and Shimizu 1985). Secondly, anthocyanins can directly remove the reactive oxygen species (ROS) (Neill and Gould 2003). Many studies have confirmed that anthocyanin accumulation in young leaves ensures the normal growth of young leaves in high-light and high-temperature environment (Zhang et al. 2016, 2018a; Zhu et al. 2016, Yu et al. 2019, 2020), which plays a role in photoprotection. However, the sequence analysis of anthocyanin synthesis-related genes in the young leaves of two tree species (i.e., Machilus chinensis and Castanopsis chinensis) in the subtropical forest has not been reported. Therefore, obtaining the anthocyaninrelated gene sequences of these two tree species has great significance to prove the importance of anthocyanins in photoprotection.

Besides, some low-molecular antioxidants synthesized by plants play an important role in preventing and alleviating photoinhibition. The responses of ascorbic acid, tocopherol, glutathione, carotenoids, flavonoids, and phenolics to stress in plants have been widely studied (Rice-Evans et al. 1997, Wang et al. 1997, Agati et al. 2007, Neill et al. 2002, Agati and Tattini 2010, Zhu et al. 2016). In the high-light environment, the balance of normal ROS production and scavenging was disturbed in plants. At this time, plants can restore this balance by increasing the contents of the antioxidants (Foyer and Noctor 2005, Foyer and Shigeoka 2011). Among them, carotenoids play an important photoprotective role by quenching triplet state chlorophyll molecules and scavenging singlet oxygen formed within the chloroplast (Young 1991). Ascorbic acid can directly quench most forms of ROS and regulate the redox state of photosynthesis (Miller et al. 2010). It has been shown that phenolics also play an important role in photoprotection during the development of woody plant leaves (Zhang et al. 2018b). In addition, heat dissipation was also one of the important protective mechanisms for plants to cope with photoinhibition (Hirotsu et al. 2004, Hu et al. 2008). At present, researchers believe that heat dissipation is the main mechanism of plant photoprotection (Endo et al. 2014). Heat dissipation, photosynthesis, and Chl fluorescence are the three ways for photosynthetic organs to utilize light energy: photosynthesis + Chl fluorescence + heat dissipation $=1$. Their relationship is competitive with each other and Chl fluorescence only accounts for a small part. Therefore, heat dissipation plays an important role in the process of photoprotection when the photosynthetic carbon assimilation rate of plants decreases under strong light stress. At present, the nonphotochemical quenching (NPQ) of Chl fluorescence is used to reflect the heat dissipation of plants.

However, the anthocyanin synthesis-related genes and internal reference gene sequences of the two species of subtropical forest used in this experiment are still unknown, so obtaining these gene sequences can play an important role in exploring the photoprotection mechanism of anthocyanins in the species of subtropical forest. At the same time, this study revealed that plants not only rely on anthocyanins as a means of photoprotection to cope with the high-light environment, but also gain photoprotection ability through other ways, such as antioxidants and NPQ. Therefore, different species take different photoprotection measures in the face of high temperature and high-light environment but their common goal is to ensure the normal growth of young leaves to maintain the stability of the population.

\section{Materials and methods}

Plant materials: Machilus chinensis and Castanopsis chinensis seedlings were collected from Dinghu Mountain

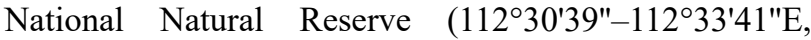
$\left.23^{\circ} 09^{\prime} 21^{\prime \prime}-23^{\circ} 11^{\prime} 30^{\prime \prime} \mathrm{N}\right)$ and were planted at the experimental site of the South China Normal University 
Biological Garden in pots. The climate conditions of the planting area are consistent with the original site, which belongs to the monsoon climate. When the seedlings survived, they were divided into two groups and each group contained ten pots. One group was grown under $100 \%$ full sunlight and the other group was grown under a black nylon shading net and only retained $30 \%$ full sunlight (Zhu et al. 2016). Regular watering, fertilizing and weeding were routine management practices performed. Five years later, the experiment began. From July to September 2015 and 2016, the experiments were carried out on leaves of different leaf positions under a different light.

Total RNA extraction and cDNA synthesis: The total RNA of $M$. chinensis and $C$. chinensis were extracted by Plant RNA Kit (Huayue Biotech, Beijing, China). Fresh leaves $(0.25 \mathrm{~g})$ were grinded to powder in a mortar in liquid nitrogen, and then immediately and thoroughly mixed with lysis solution. The remaining extraction steps followed the instructions of the kit to extract total RNA from the sample, while 1\% agarose gel electrophoresis was used to identify the integrity of total RNA. The purity and concentration of RNA were detected by measuring the optical density (OD) at 260-280 nm using a Nanodrop ND-2000C spectrophotometer (Thermo Scientific). The first-strand cDNA was synthesized by oligo (dT) 18 primer and the reverse transcriptase of Takara's $M$ - $M L V$-Reverse. The total RNA of $1 \mu \mathrm{g}$ was used for each retrotranscription.

Gene cloning and sequencing: Genes were cloned and sequenced for partial fragments of $C H S, D F R$, $A N S$, and TUB genes of $M$. chinensis. First of all, we searched GenBank of NCBI website to find out whether there were these gene sequences of species close to the phylogeny of $M$. chinensis, and found that there were no relevant results in the genus of Machilus nees, so we searched for the related gene sequences of Lauraceae in the upper level. We found that Persea americana had the sequences of these genes, so we used these gene sequences to clone the genes of $M$. chinensis. Cloning primers were designed with the related genes of $P$. americana, and the cDNA of young leaves of $M$. chinensis was amplified by PCR. If the electrophoretic band of the PCR product was a single band of the target length, the product was sent to the company for sequencing. The sequencing results were aligned by $B L A S T$ on NCBI website to determine whether these sequences were expected. If there was a high similarity between the sequences of these genes and those of other species, we can expect that the sequences are of $C H S, D F R, A N S$, and TUB of M. chinensis.

Gene were cloned and sequenced for partial fragments of CHS, DFR, ANS, and TUB genes of C. chinensis. We found that Castanea sativa was closely related to C. chinensis in GenBank of NCBI website, so we could directly use $C H S, D F R, A N S$, and $T U B$ gene sequences of $C$. sativa to design clone primers for the corresponding genes. And the non-full-length sequences of these genes were obtained directly by electronic cloning. In order to prove the correctness of these gene sequences, the similarity between these gene sequences and another closely related species of Castanopsis fissa was compared with DNAMAN8 software. At the same time, these sequences were aligned by BLAST on NCBI website. If there is a high similarity between the comparison results and other gene sequences of non-related species, it can be determined that these gene sequences are sequences of C. chinensis.

Primer design and synthesis: In order to design the clone primers for partial fragments of $C H S, D F R, A N S$, and $T U B$ genes of $M$. chinensis and $C$. chinensis, the known $C H S$, $D F R$, and $A N S$ gene sequences were found in GenBank at the NCBI website. These $C H S, D F R$, and $A N S$ gene sequences of the two tree species in this experiment were compared using MegAlign software to find conserved regions. Then, the highest homology nucleotide sequence was used as a template, and primers were designed using DNAMAN software.

Gene expression analysis: The relative expression levels of $C H S, D F R$, and $A N S$ genes were analyzed by $S Y B R$ Premix Ex TaqTM II Kit (Takara) and ABI7500 Real-Time PCR System (Applied Biosystems, Foster City, USA). The $T U B$ was the reference gene. The gene expression reaction system and reaction temperature were designed according to Zhang et al. (2016). The relative expression of $C H S, D F R$, and $A N S$ genes in the leaves of two tree species under two different light conditions was calculated by $2^{-\Delta \Delta \mathrm{CT}}$ method (Livak and Schmittgen 2001). Five biological samples were used.

Anthocyanin content: Anthocyanins were extracted by $1 \%$ methanol: $\mathrm{HCl}(99: 1, \mathrm{v} / \mathrm{v})$ solution. First of all, we wiped the selected plant leaves with distilled water, weighed $0.2 \mathrm{~g}$ of fresh plant leaves with main veins removed, and put them in a mortar containing $2 \mathrm{~mL}$ of $1 \%$ methanol: $\mathrm{HCl}$ solution, and ground them into a homogenate, and transferred to a centrifuge tube. The solution was fully soaked at $4^{\circ} \mathrm{C}$ in dark for $24 \mathrm{~h}$. When the anthocyanins were completely extracted, an equal volume of chloroform and $1 \mathrm{~mL}$ of distilled water were added. At this time, the solution was divided into two layers. Chl was dissolved in the lower chloroform phase and anthocyanins were dissolved in the upper methanol-water phase. The upper solution was absorbed for measurement, and then spectral absorption scanning was performed with a $U V-2450$ spectrophotometer (Shimadzu, Tokyo, Japan) at the wavelength of 400-700 $\mathrm{nm}$. The anthocyanin content was calculated using the method described by Reddy et al. (1995).

Flavonoids, phenolics, and total antioxidant capacity: Fresh leaves of $0.1 \mathrm{~g}$ with the main veins removed were placed in a labeled centrifuge tube with $3 \mathrm{~mL}$ of $95 \%$ methanol solution, and then they were soaked overnight in a refrigerator at $4^{\circ} \mathrm{C}$ to extract flavonoids and phenolics. The determination method of flavonoids was slightly 
modified according to the method of Heimler et al. (2005). The phenolic contents of the plant leaves were determined according to the methods of Ainsworth and Gillespie (2007). The determination method of total antioxidant capacity was carried out according to Saha et al. (2008). The total antioxidant capacity in the sample was determined by monitoring the scavenging amount of 1,1-diphenyl2-picrylhydrazyl (DPPH) radicals in the extract. The total antioxidant capacity of different leaves was expressed in nmol of DPPH scavenged per unit mass.

Chl fluorescence after high-light stress: Healthy young leaves and mature leaves were selected, and their petioles were immersed in water immediately after the detachment. Then, the leaves were allowed to fully adapt to the dark, and then the leaves were treated with $1,800 \mu \mathrm{mol}$ (photon) $\mathrm{m}^{-2} \mathrm{~s}^{-1}$ of white light for $60 \mathrm{~min}$ at a temperature of $30^{\circ} \mathrm{C}$. After high-light treatment, the leaves returned to the dark environment to recover for $60 \mathrm{~min}$. During the treatment, the change of the maximum photochemical efficiency $\left(\mathrm{F}_{\mathrm{v}} / \mathrm{F}_{\mathrm{m}}\right)$ was measured with a PAM 2100 portable fluorometer (Waltz, Germany) every $20 \mathrm{~min}$. In the process of high-light treatment, the petiole was inserted in water to prevent water loss of leaves (Hughes et al. 2005).

Determination of $\mathbf{C h l}$ fluorescence: Chl fluorescence was measured by $\mathrm{Chl}$ fluorescence imaging system $C F I$ (Technologica, UK). The young and mature leaves after dark adaptation for 30 min were placed on CFI platform of $\mathrm{Chl}$ fluorescence imaging system to measure $\mathrm{Chl}$ fluorescence. The response curve of $\mathrm{Chl}$ fluorescence to light intensity was measured by the instrument's own blue light source. Subsequently, the samples were irradiated with a measuring light with an intensity lesser than $0.05 \mu$ mol(photon) $\mathrm{m}^{-2} \mathrm{~s}^{-1}$; this was used to obtain the initial fluorescence $\left(\mathrm{F}_{0}\right)$ and then a pulse of 6,000 $\mu$ mol(photon) $\mathrm{m}^{-2} \mathrm{~s}^{-1}$ of saturated light was used to obtain the maximum fluorescence $\left(\mathrm{F}_{\mathrm{m}}\right)$. The gradient settings of PPFD were 15, 30, 60, 100, 200, 400, 600, 800; 1,000; and $1,200 \mu \mathrm{mo}$ (photon) $\mathrm{m}^{-2} \mathrm{~s}^{-1}$, respectively. The adaptation time for each light intensity was $120 \mathrm{~s}$. The $\mathrm{F}_{0}$ and $\mathrm{F}_{\mathrm{m}}{ }^{\prime}$ of the light-adapted state at each PPFD were recorded. The NPQ was calculated using the following equation: $\mathrm{NPQ}=\left(\mathrm{F}_{\mathrm{m}} / \mathrm{F}_{\mathrm{m}}{ }^{\prime}\right)-1$ (Bilger and Björkman 1990).

Statistical analysis: The statistical analysis of the data was performed using IBM SPSS Statistics SPSS 19.0 software (IBM, NY, USA). Duncan's multiple comparison method was used to test differences in physiological indexes between different leaf positions of plants. The significance level was $P<0.05$. SigmaPlot 12.5 software (USA) was used for drawing. The data were presented as the mean \pm standard error (SE).

\section{Results}

RNA extraction and sample quality testing: The total RNA of M. chinensis and C. chinensis leaves were detected by $1 \%$ agarose gel electrophoresis for RNA quality (Fig. 1). The total RNA of the samples of both $M$. chinensis and $C$. chinensis leaves showed neat rRNA bands (Fig. 1), both containing $28 \mathrm{~S}$ and $18 \mathrm{~S}$ bands with clear brightness, relatively good RNA integrity, and no obvious degradation. The Nanodrop ND-2000C spectrophotometer showed that the RNA $\mathrm{OD}_{260 \mathrm{~nm}} / \mathrm{OD}_{280 \mathrm{~nm}}$ of each sample was between 1.80-2.00, indicating that the RNA complied to the requirements of subsequent experiments. Fig. $2 A$ shows the primer sequences of $C H S, D F R, A N S$, and TUB genes for $M$. chinensis. The reverse transcription cDNAs from the leaves of $M$. chinensis were used as templates and amplified by PCR, and the PCR products of $C H S, D F R, A N S$, and $T U B$ were analyzed by agarose gel electrophoresis (Fig. $2 B-D$ ). We found that the electrophoresis images of the amplified products were all single bands, and the product length were all between 500-750 bp, which was consistent with the predicted results. The purified products were sent to Majorbio (Shanghai, China) for sequencing.

Homologous alignment of the CHS, DFR, and $A N S$ partial nucleotide sequences: After sequencing, a homologous alignment of the $C H S, D F R, A N S$, and $T U B$ partial nucleotide sequences of $M$. chinensis and $C$. chinensis was performed via nucleotide Blastn and Blastx on the NCBI website to examine their homology to the partial nucleotide sequences of these genes in known plants. It was found that they are very similar to some nucleotide sequences of these genes in known species (Table 1). Some nucleotide sequences of the cloned $M$. chinensis $C H S$ gene had the highest similarity with Persea americana (97.3\%), and $C$. chinensis had the highest similarity with Quercus lobata $(97.2 \%)$. Part of the nucleotide sequence of the cloned $M$. chinensis DFR gene had the highest similarity with Medicago truncatula (75.2\%), and C. chinensis had the highest similarity with Quercus suber (97.5\%). Some nucleotide sequences of the cloned $M$. chinensis ANS gene had the highest similarity with Elaeis guineensis (78.9\%), and $C$. chinensis had the highest similarity with Quercus lobata $(96.9 \%)$. Some nucleotide sequences of the cloned M. chinensis $T U B$ gene had the highest similarity with Litsea cubeba $(97.9 \%)$, and $C$. chinensis had the highest similarity with Castanea mollissima (99.6\%). Therefore, it can be confirmed that these sequences obtained by cloning

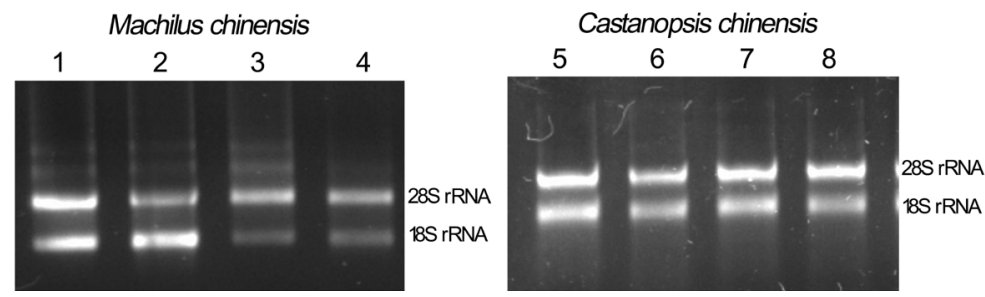

Fig. 1. Agarose gel electrophoresis image of total RNA extracted products from leaves of Machilus chinensis (1-4) and Castanopsis chinensis (5-8). 


\begin{tabular}{cccc}
\hline Gene & Forward primer sequence & Reverse primer sequence & Product length [bp] \\
\hline CHS & ACTACTACTTCGGATCACCA & ACTTCCCTCAAATGTCCATC & 670 \\
DFR & AAGCCCACAATAAATGGAGT & TGTAGGGATGTTGTATTCCG & 535 \\
ANS & TATCCTGACCTTCTCCTTGT & AAGGCATTTTTCGATCTTCC & 600 \\
TUB & TTCCATCGCCTAAAGTTTCT & GAGGTAGAGTTGCCAATGAA & 609 \\
\hline
\end{tabular}

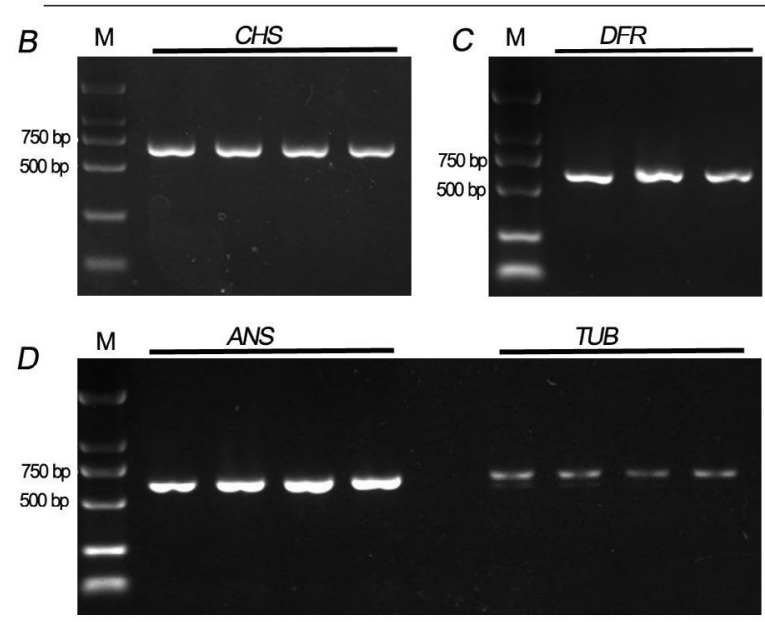

Fig. 2. The chalcone synthase (CHS), dihydroflavonol 4-reductase (DFR), anthocyanin synthase (ANS), and $\beta$-tubulin (TUB) primer sequences for Machilus chinensis $(A)$; the agarose gel electrophoresis images of PCR cloned products of $C H S, D F R, A N S$, and TUB genes of Machilus chinensis $(B-D)$.

Table 1. Comparison results of partial nucleotide sequences of chalcone synthase (CHS), dihydroflavonol 4-reductase (DFR), and anthocyanin synthase (ANS) genes of Machilus chinensis and Castanopsis chinensis with CHS, DFR, and ANS genes of other known species.

\begin{tabular}{|c|c|c|c|c|}
\hline Gene & Species & Species & GeneBank accession & Identity [\%] \\
\hline \multirow[t]{6}{*}{ CHS } & \multirow[t]{3}{*}{ Machilus chinensis } & Persea americana & EU335633 & 97.30 \\
\hline & & Vaccinium angustifolium & MK333526 & 84.44 \\
\hline & & Vaccinium ashei & AB694903 & 84.27 \\
\hline & \multirow[t]{3}{*}{ Castanopsis chinensis } & Quercus lobata & XM_031118777 & 97.21 \\
\hline & & Quercus suber & XM_024058423 & 97.04 \\
\hline & & Juglans regia & XM_018961711 & 86.27 \\
\hline \multirow[t]{6}{*}{$D F R$} & \multirow[t]{3}{*}{ Machilus chinensis } & Medicago truncatula & XM_013610680 & 75.16 \\
\hline & & Prosopis alba & XM_028918146 & 75.00 \\
\hline & & Lotus japonicus & AB162109 & 74.25 \\
\hline & \multirow[t]{3}{*}{ Castanopsis chinensis } & Quercus suber & XM_024016259 & 97.50 \\
\hline & & Quercus lobata & XM_031094690 & 97.22 \\
\hline & & Carya illinoinensis & MH613770 & 84.51 \\
\hline \multirow[t]{6}{*}{$A N S$} & \multirow[t]{3}{*}{ Machilus chinensis } & Elaeis guineensis & XM_010911553 & 78.93 \\
\hline & & Strelitzia reginae & $\mathrm{KC} 484623$ & 78.89 \\
\hline & & Phoenix dactylifera & XM_008785360 & 78.49 \\
\hline & \multirow[t]{3}{*}{ Castanopsis chinensis } & Quercus lobata & XM_031092627 & 96.91 \\
\hline & & Quercus suber & XM_024021064 & 96.82 \\
\hline & & Juglans regia & XM_018991659 & 85.47 \\
\hline
\end{tabular}

were partial nucleotide sequences of $C H S, D F R, A N S$, and $T U B$ genes of $M$. chinensis and $C$. chinensis, respectively (Figs. 1S-8S, supplement).

Analysis of the melting curve of the CHS, DFR, ANS, and $\boldsymbol{T U B}$ gene in qRT-PCR: After obtaining the $C H S$, $D F R, A N S$, and TUB gene sequences of $M$. chinensis and
C. chinensis, according to the primer design principle of fluorescence quantitative PCR, NCBI online primer design website was used to design fluorescence quantitative PCR primers for the corresponding genes (Fig. 13S $A, B$; supplement). The fluorescence quantitative PCR primers of $C H S, D F R, A N S$, and TUB of M. chinensis and $C$. chinensis were used to carry out PCR. The electrophoretic 
results showed that the amplified products were all single bands with the expected length (Fig. 3). The qRT-PCR was carried out using the cDNA of $M$. chinensis and C. chinensis leaves as templates. The results showed that the fluorescence quantitative PCR melting curves of $C H S, D F R, A N S$, and $T U B$ genes of the two species were all single peaks without messy peaks, and the single peaks were between $82-88^{\circ} \mathrm{C}$, indicating that there was no nonspecific amplification and no primer dimers for each gene primer (Fig. 13SC,D; supplement). The primers of each gene had the good specificity. The results were accurate and reliable, in line with qRT-PCR standards, and could be used for subsequent experiments.

Anthocyanin contents and the expression of $\mathrm{CHS}, \mathrm{DFR}$, and $\boldsymbol{A N S}$ : The young leaves of $M$. chinensis showed that there was no anthocyanin accumulation under both $100 \%$ and $30 \%$ full sunlight. The expression of some key enzyme genes of anthocyanin synthesis pathway ( $C H S, D F R$, and $A N S$ ) in the young leaves was relatively low (Fig. $4 A, C, E)$. On the contrary, we found in the young leaves of $C$. chinensis that there was anthocyanin accumulation under both light intensities. Among them, the anthocyanin content in the leaves under $100 \%$ full sunlight was significantly higher than that under $30 \%$ full sunlight, and the anthocyanin content in the first young leaves was the highest, followed by the second young leaves, while no anthocyanin accumulation was observed in the mature leaves (Fig. 4B). Besides, we found from the expression levels of the related enzyme genes in anthocyanin synthesis pathway in young leaves of $C$. chinensis that the expression levels of $C H S, D F R$, and $A N S$ genes under $100 \%$ full sunlight was significantly higher than that under $30 \%$ full sunlight, with the highest expression levels in the first young leaves, followed by the second young leaves, and the lowest expression in the mature leaves (Fig. 4D,F). This was consistent with the accumulation trend of C. chinensis anthocyanins.

Flavonoid content, phenolic contents, and total antioxidant capacity: At the two light intensities, the flavonoid content, phenolic contents, and total antioxidant capacity of the young leaves of $M$. chinensis and $C$. chinensis were significantly higher than those of the mature leaves (Fig. 5). The first young leaves contained the highest amounts, the second young leaves followed, and the mature leaves had the least amounts. In the leaves of $M$. chinensis and C. chinensis, the flavonoid content, phenolic contents, and total antioxidant capacity of young leaves in the same leaf position under $100 \%$ full sunlight environment were higher than those of young leaves in the same leaf position under $30 \%$ full sunlight environment. At the same leaf position, whether under $100 \%$ full sunlight or $30 \%$ full sunlight, the flavonoid content, phenolic contents, and total antioxidant capacity of $M$. chinensis young leaves were higher than that of $C$. chinensis.

Chl fluorescence: The results of light curve analysis showed that the NPQ of young leaves of $M$. chinensis and $C$. chinensis were significantly higher than that of the mature leaves. The first young leaves were the highest followed by the second young leaves and the mature leaves were the smallest. The NPQ value of leaves under $100 \%$ full sunlight was higher than that under $30 \%$ full sunlight. When comparing the NPQ differences between $M$. chinensis and $C$. chinensis species, we found that the NPQ value of $M$. chinensis leaves was higher than that of $C$. chinensis leaves under both $100 \%$ and $30 \%$ full sunlight (Fig. 6A,B). Under the high-light treatment of $1,800 \mu \mathrm{mol}$ (photon) $\mathrm{m}^{-2} \mathrm{~s}^{-1}$, both $M$. chinensis and C. chinensis were stressed, and the $\mathrm{F}_{\mathrm{v}} / \mathrm{F}_{\mathrm{m}}$ of their leaves decreased significantly (Fig. $6 C, D$ ). The decrease of $\mathrm{F}_{\mathrm{v}} / \mathrm{F}_{\mathrm{m}}$ in young leaves growing under $30 \%$ full sunlight was higher than that under $100 \%$ full sunlight in both species. Under the same light conditions, the $\mathrm{F}_{\mathrm{v}} / \mathrm{F}_{\mathrm{m}}$ change range of $C$. chinensis leaves after high-light treatment was smaller than that of $M$. chinensis.

\section{Discussion}

Anthocyanins are derivatives of benzopyran and are the most abundant water-soluble pigments in many plant species (Oancea and Oprean 2011). Anthocyanin synthesis is regulated by a series of enzyme genes, in which structural genes directly encode the enzymes needed for anthocyanin synthesis, and their expression is directly related to anthocyanin accumulation (Nakatsuka et al. 2005a). CHS is the first key enzyme or rate-limiting enzyme in the anthocyanin synthesis pathway (Deng et al. 2014). Its expression is directly related to plant color. $D F R$ is a necessary structural gene for plant leaf coloration. ANS is also known as dioxygenase (Tanaka et al. 2008). It mainly catalyzes the transformation of colorless anthocyanins into various colored anthocyanins, which is a rate-limiting enzyme for the anthocyanin synthesis. The expression of $A N S$ gene is positively correlated with the anthocyanin content (Welford et al. 2005). Therefore, it is important to study the expression of these
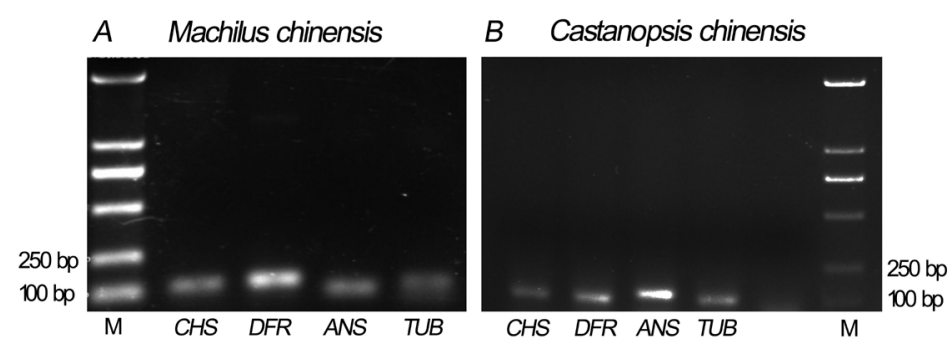

Fig. 3. Agarose gel electrophoresis patterns of PCR products of chalcone synthase (CHS), dihydroflavonol 4-reductase (DFR), anthocyanin synthase (ANS), and $\beta$-tubulin (TUB) genes of Machilus chinensis and Castanopsis chinensis. 


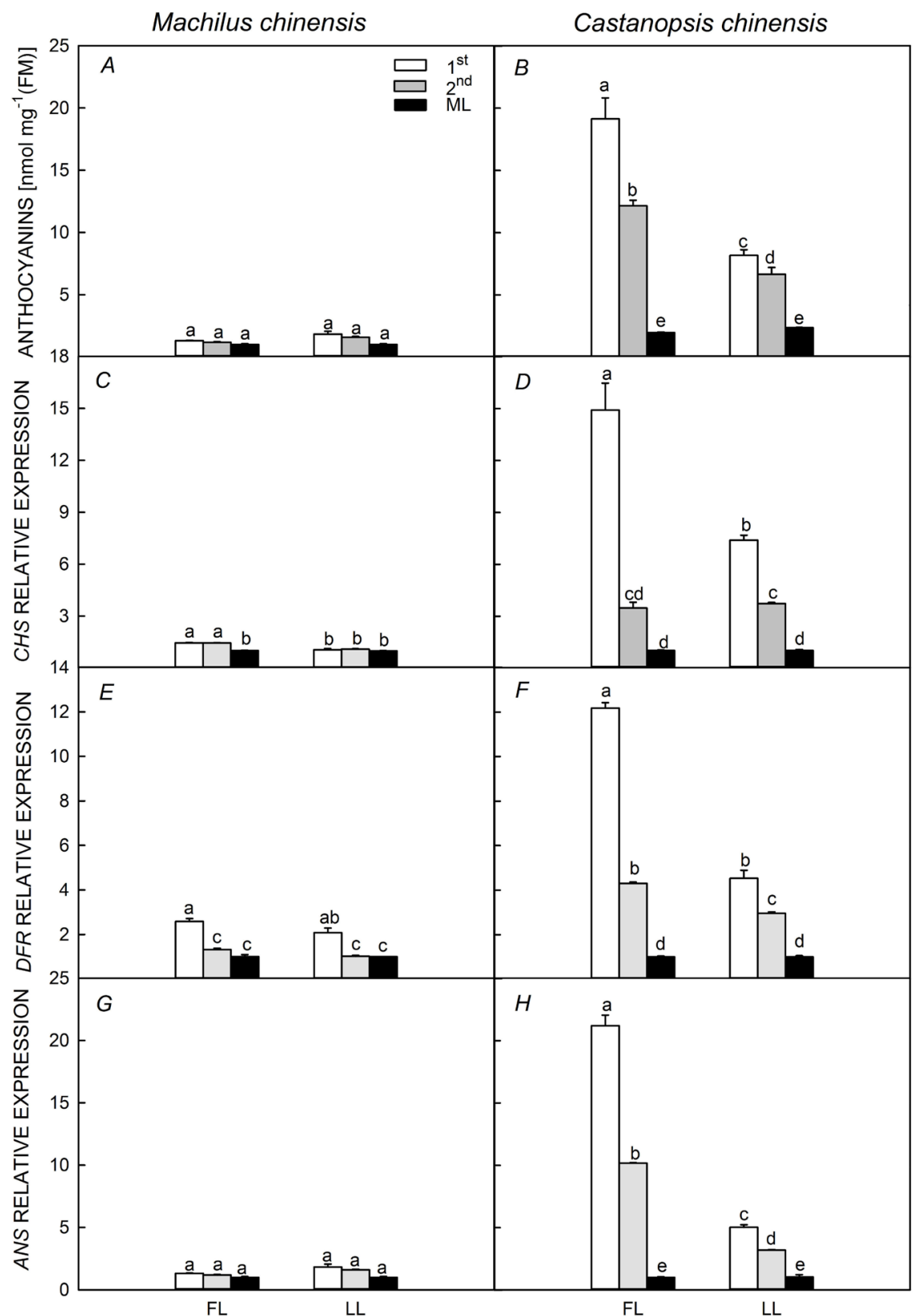

Fig. 4. Anthocyanin content $(A, B)$, chalcone synthase $(C H S)(C, D)$, dihydroflavonol 4-reductase (DFR), and anthocyanin synthase (ANS) $(G, H)$ gene relative expression in different leaf positions of Machilus chinensis and Castanopsis chinensis under different light conditions. Data are presented as the means $\pm \mathrm{SE}(n=5)$; different letters represent statistically significant differences $(P<0.05) .1^{\text {st }}$ - the first young leaves; $2^{\text {nd }}-$ the second young leaves; $\mathrm{ML}$ - mature leaves; FL - 100\% full sunlight; $\mathrm{LL}-30 \%$ full sunlight. three genes. They can prove the role of anthocyanins in photoprotection of the young leaves of subtropical forest plants. Total RNA of the M. chinensis and C. chinensis leaves was extracted (Fig. 1) and reverse transcribed to cDNA as templates for PCR amplification. After verifying by agarose gel electrophoresis (Fig. $2 B-D$ ), PCR product was purified and sent to Majorbio (Shanghai, China) for sequencing to obtain part of the nucleotide sequences of the target genes (Figs. 1S-8S). Because of the discovery of Castanea sativa which is closely related to C. chinensis, the nucleotide sequences of the target genes were directly obtained by the method of electronic cloning. In order to verify the accuracy, we compared the similarity with another closely related species Castanopsis fissa and found that these genes are very similar (Figs. 9S-11S, supplement). After that, we compared the homology of some nucleotide sequences of these genes in known plants to determine whether the cloned sequences were partial sequences of $C H S, D F R$, and $A N S$ genes of subtropical tree species (Table 1). At last, some nucleotide sequences of the target genes were sequenced to design qRT-PCR primers (Fig. 13S $A$ ), and the fluorescent quantitative PCR primers of these genes were analyzed by common PCR (Fig. 3). The gene expression was verified by qRT-PCR technology (Fig. 4). Finally, it was determined that CHS, $D F R$, and $A N S$ genes belong to the two tree species in the subtropical forest.

High temperature and high-light environment in summer can severely inhibit photosynthesis of plants (Zhang et al. 2018a). However, the young leaves at the top of the 


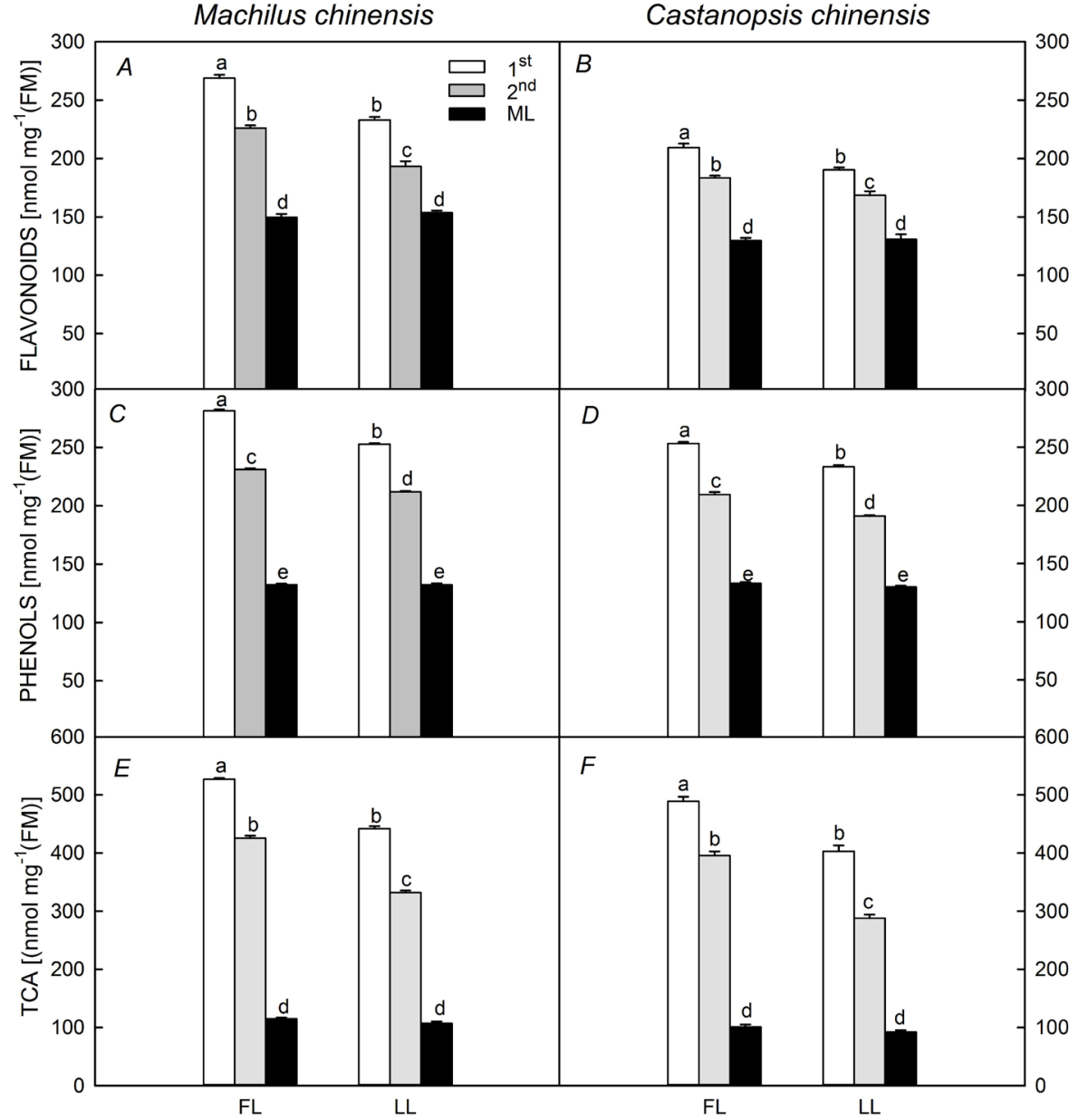

Fig. 5. Flavonoid content $(A, B)$, phenolics content $(C, D)$, and total antioxidant capacity (TCA) $(E, F)$ of different leaf positions of Machilus chinensis and Castanopsis chinensis under different light conditions. Data are presented as the means $\pm \mathrm{SE}(n=5)$; different letters represent statistically significant differences $(P<0.05)$. $1^{\text {st }}-$ the first young leaves; $2^{\text {nd }}-$ the second young leaves; ML - mature leaves; FL - 100\% full sunlight; LL $-30 \%$ full sunlight. canopy have various defense mechanisms to deal with the high temperature and high-light environment (Zhang et al. 2016). In order to make up for the mismatch between photosynthetic energy utilization ability and light energy capture ability, these young leaves accumulate a large number of anthocyanins, synthesize a large number of antioxidants, and enhance their heat dissipation to obtain photoprotection ability. Anthocyanins play an important role in the process of photoprotection. Hughes et al. (2007) believed that the accumulation time of anthocyanins was strongly coupled with the development process of leaves, and anthocyanins could play a role in protecting tissues until its photosynthetic mechanism was mature. This provides strong support for the photoprotective effect of anthocyanins in developing leaves. Many studies have shown that anthocyanins accumulated in plant leaves mediate photoprotection mainly by intercepting light energy (Gould et al. 1995, Neill and Gould 2003, Hughes et al. 2005, Tucić et al. 2009, Zhang et al. 2010). Anthocyanins can absorb yellow, green, and blue light in visible spectrum to change light quality and reduce light intensity (Feild et al. 2001, Karageorgou and Manetas 2006), to achieve the purpose of shielding from excess light energy for photosynthetic apparatus. Secondly, anthocyanins can directly remove ROS. Neill and Gould (2003) proved that the colorless and red tautomers of cyanidin glycoside can clear ROS produced by chloroplast suspension under mild stress. Besides, Zeng et al. (2010) also reached the same conclusion; anthocyanins have a very strong ROS-scavenging ability. Therefore, anthocyanin accumulation in young leaves of plants can be used not only as a light attenuator, but also as an antioxidant.

The decrease of $\mathrm{F}_{\mathrm{v}} / \mathrm{F}_{\mathrm{m}}$ of $\mathrm{Chl}$ fluorescence in plants is a significant feature of photoinhibition of photosynthesis (Demmig-Adams and Adams 1992). The $F_{v} / F_{m}$ ratio of healthy plants is usually stable but when are plant exposed to stress, the $F_{v} / F_{m}$ ratio decreases significantly. It is a simple method to study the tolerance of different leaves to light stress by treating leaves with high light, and then measuring the change of $F_{v} / F_{m}$ ratio before and after treatment (Force et al. 2003, Schubert et al. 2006). After high-light stress, the $F_{\mathrm{v}} / \mathrm{F}_{\mathrm{m}}$ ratio of red young leaves of $C$. chinensis decreased less than that of green young leaves of $M$. chinensis (Fig. 6C,D). This indicated that anthocyanins formed a light barrier on the surface of plant leaves, weakened the light energy entering the leaves, and delayed the effect of light stress on plant leaves. Besides anthocyanins, some low-molecular antioxidants synthesized in plants also play an important role in the process of photoprotection. Some young leaves of plants can change the synthesis of secondary metabolites to adapt to the high-light environment. Zhang et al. (2018b) 

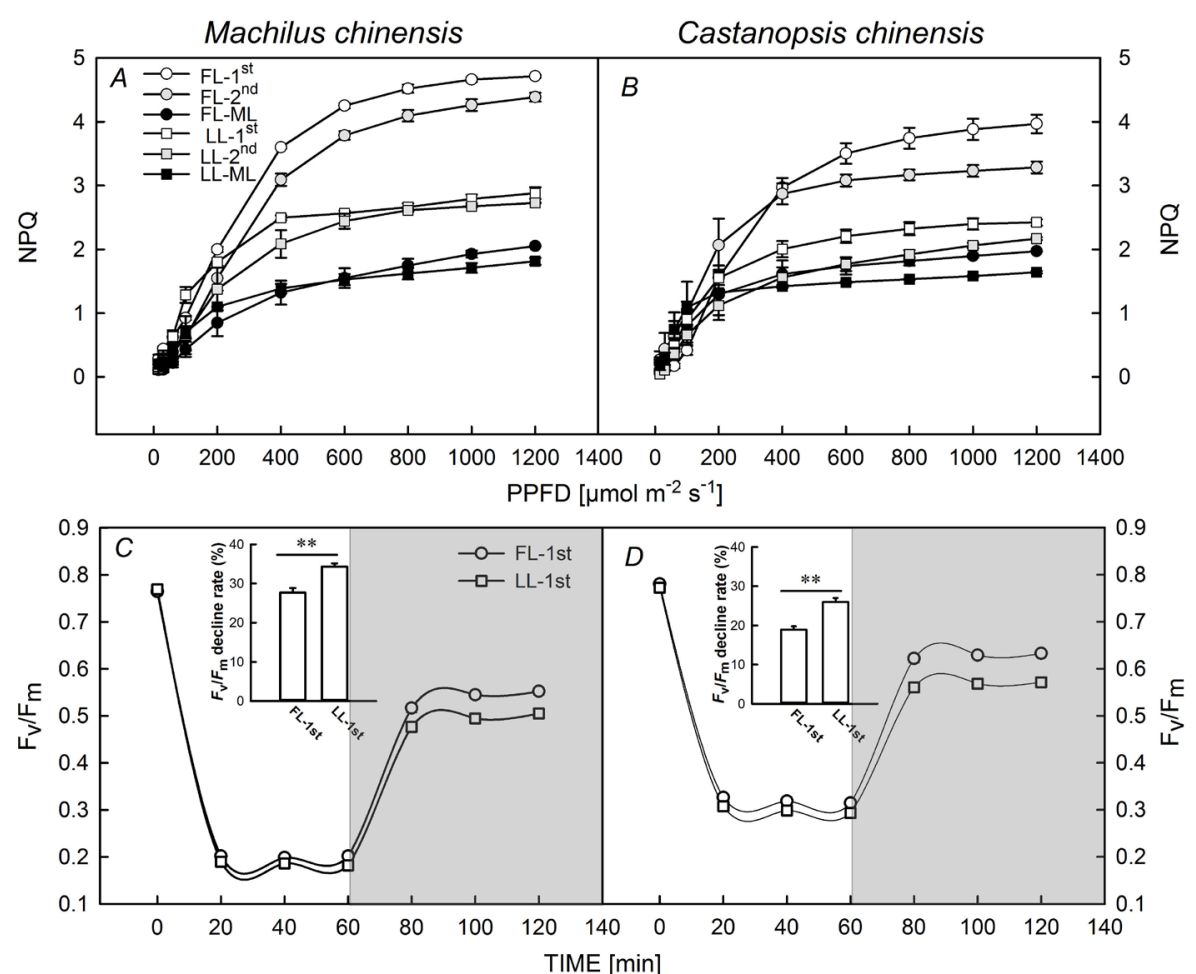

Fig. 6. The response curves of nonphotochemical quenching (NPQ) $(A, B)$ and the change of maximal quantum yield of PSII photochemistry $\left(\mathrm{F}_{\mathrm{v}} / \mathrm{F}_{\mathrm{m}}\right)(C, D)$ after highlight treatment of different leaf positions of Machilus chinensis and Castanopsis chinensis under different light conditions; white areas represent high-light treatment; gray areas represent dark treatment. Data are presented as the means $\pm \operatorname{SE}(n=5) \cdot 1^{\text {st }}-$ the first young leaves; $2^{\text {nd }}-$ the second young leaves; $\mathrm{ML}$ - mature leaves; $\mathrm{FL}-100 \%$ full sunlight; $\mathrm{LL}-30 \%$ full sunlight. confirmed that young leaves of plants can adapt to different growth light intensity by improving the synthesis of secondary metabolites such as flavonoids and phenolics through physiological and transcriptome analysis results. In this study, we found that the flavonoids and phenolics contents in young leaves containing anthocyanins were also high but the content of flavonoids and phenolics in young leaves without anthocyanin accumulation was higher (Fig. 5A-D). It was shown that anthocyanins cannot completely shield and eliminate the adverse effects of excess light energy when the light intensity is high. At this time, plants increase contents of these antioxidants to achieve the best photoprotection. At the same time, it was also proved that these low-molecular antioxidants are the most basic and key means of photoprotection for plants. Increasing heat dissipation capacity is another defense method of plants when absorbing excessive light energy. Previous studies have found that plants exposed to high light for a short time dissipate the excess light energy by increasing NPQ (Mai et al. 2010). The corresponding curves of fluorescence showed that the NPQ value of red young leaves of $C$. chinensis was lower than that of green young leaves of $M$. chinensis (Fig. $6 A, B$ ). It means that NPQ can balance the deficiency of anthocyanin-mediated photoprotection in high-light environment. Within a certain range of high light, NPQ of plant leaves increased with the increase of light intensity, which can also explain why the NPQ changes and anthocyanin content changes were of the opposite trend.

Conclusion: The young leaves of forest species in subtropical forests can grow normally under high-light environment. But different plants adopt different photoprotection means under high light. The young leaves of
C. chinensis accumulate anthocyanins to intercept light energy, and when the light intensity is too high, they accumulate higher amounts of antioxidants and increase NPQ to achieve the photoprotection. Since the young leaves of $M$. chinensis did not accumulate anthocyanins under the high-light stress, they concentrate all resources on the accumulation of antioxidants and the increase of NPQ, thus forming the strong photoprotection potential. In conclusion, the accumulation of anthocyanins, the synthesis of antioxidants, and the improvement of NPQ together mediate the positive photoprotection of young leaves on photoadaptation, which provides a strong guarantee for the successful development of young leaves in different light intensity environments. In addition, sequencing and comparing the genes related to anthocyanin biosynthesis in young leaves of tree species in subtropical forest was important to reveal the photoprotective role of anthocyanins in young leaves under high-light environment.

\section{References}

Agati G., Matteini P., Goti A., Tattini M.: Chloroplast-located flavonoids can scavenge singlet oxygen. - New Phytol. 174: $77-89,2007$.

Agati G., Tattini M.: Multiple functional roles of flavonoids in photoprotection. - New Phytol. 186: 786-793, 2010.

Ainsworth E.A., Gillespie K.M.: Estimation of total phenolic content and other oxidation substrates in plant tissues using Folin-Ciocalteu reagent. - Nat. Protoc. 2: 875-877, 2007.

Bilger W., Björkman O.: Role of the xanthophyll cycle in photoprotection elucidated by measurements of light-induced absorbance changes, fluorescence and photosynthesis in leaves of Hedera canariensis. - Photosynth. Res. 25: 173185,1990

Chalker-Scott L.: Environmental significance of anthocyanins in 
plant stress responses. - Photochem. Photobiol. 70: 1-9, 1999.

Demmig-Adams B., Adams III W.W.: Photoprotection and other responses of plants to high light stress. - Annu. Rev. Plant Phys. 43: 599-626, 1992.

Deng X.B., Bashandy H., Ainasoja M. et al.: Functional diversification of duplicated chalcone synthase genes in anthocyanin biosynthesis of Gerbera hybrida. - New Phytol. 201: 1469-1483, 2014.

Dominy N.J., Lucas P.W., Ramsden L.W. et al.: Why are young leaves red? - Oikos 98: 163-176, 2002.

Endo T., Uebayashi N., Ishida S. et al.: Light energy allocation at PSII under field light conditions: How much energy is lost in NPQ-associated dissipation? - Plant Physiol. Bioch. 81: 115220, 2014.

Faria T., Silvério D., Breia E. et al.: Differences in the response of carbon assimilation to summer stress (water deficits, high light and temperature) in four Mediterranean tree species. Physiol. Plantarum 102: 419-428, 1998.

Feild T.S., Lee D.W., Holbrook N.M.: Why leaves turn red in autumn. The role of anthocyanins in senescing leaves of redosier dogwood. - Plant Physiol. 127: 566-574, 2001.

Force L., Critchley C., van Rensen J.J.S.: New fluorescence parameters for monitoring photosynthesis in plants. Photosynth. Res. 78: 17, 2003.

Foyer C.H., Noctor G.: Oxidant and antioxidant signalling in plants: a reevaluation of the concept of oxidative stress in a physiological context. - Plant Cell Environ. 28: 1056-1071, 2005.

Foyer C.H., Shigeoka S.: Understanding oxidative stress and antioxidant functions to enhance photosynthesis. - Plant Physiol. 155: 93-100, 2011

Gould K.S., Kuhn D.N., Lee D.W., Oberbauer S.F.: Why leaves are sometimes red. - Nature 378: 241-242, 1995.

Gould K.S., Markham K.R., Smith R.H, Goris J.J.: Functional role of anthocyanins in the leaves of Quintinia serrata A. Cunn. - J. Exp. Bot. 51: 1107-1115, 2000.

Heimler D., Vignolini P., Dini M.G., Romani A.: Rapid tests to assess the antioxidant activity of Phaseolus vulgaris L. dry beans. - J. Agr. Food Chem. 53: 3053-3056, 2005.

Hirotsu N., Makino A., Ushio A., Mae T.: Changes in the thermal dissipation and the electron flow in the water-water cycle in rice grown under conditions of physiologically low temperature. - Plant Cell Physiol. 45: 635-644, 2004.

Hooijmaijers C.A.M., Gould K.S.: Photoprotective pigments in red and green gametophytes of two New Zealand liverworts. New Zeal. J. Bot. 45: 451-461, 2007.

Hu W.H., Song X.S., Shi K. et al.: Changes in electron transport, superoxide dismutase and ascorbate peroxidase isoenzymes in chloroplasts and mitochondria of cucumber leaves as influenced by chilling. - Photosynthetica 46: 581-588, 2008.

Hughes N.M., Morley C.B., Smith W.K.: Coordination of anthocyanin decline and photosynthetic maturation in juvenile leaves of three deciduous tree species. - New Phytol. 175: 675-685, 2007.

Hughes N.M., Neufeld H.S., Burkey K.O.: Functional role of anthocyanins in high-light winter leaves of the evergreen herb Galax urceolata. - New Phytol. 168: 575-587, 2005.

Karageorgou P., Manetas Y.: The importance of being red when young: anthocyanins and the protection of young leaves of Quercus coccifera from insect herbivory and excess light. Tree Physiol. 26: 613-621, 2006.

Kato M., Shimizu S.: Chlorophyll metabolism in higher plants. VI. Involvement of peroxidase in chlorophyll degradation. Plant Cell Physiol. 26: 1291-1301, 1985.

Kong J.M., Chia L.S., Goh N.K. et al.: Analysis and biological activities of anthocyanins. - Phytochemistry 64: 923-933,
2003.

Krause G.H., Virgo A., Winter K.: High susceptibility to photoinhibition of young leaves of tropical forest trees. Planta 197: 583-591, 1995

Kunz S., Burkhardt G., Becker H.: Riccionidins $A$ and $\mathrm{B}$, anthocyanidins from the cell walls of the liverwort Ricciocarpos natans. - Phytochemistry 35: 233-235, 1994.

Lee D.W., Collins T.M.: Phylogenetic and ontogenetic influences on the distribution of anthocyanins and betacyanins in leaves of tropical plants. - Int. J. Plant Sci. 162: 1141-1153, 2001.

Livak K.J., Schmittgen T.D.: Analysis of relative gene expression data using real-time quantitative PCR and the $2^{-\triangle \Delta C T}$ method. - Methods 25: 402-408, 2001.

Mai J., Herbette S., Vandame M. et al.: Contrasting strategies to cope with chilling stress among clones of a tropical tree, Hevea brasiliensis. - Tree Physiol. 30: 1391-1402, 2010.

Miller G., Suzuki N., Ciftci-Yilmaz S., Mittler R.: Reactive oxygen species homeostasis and signalling during drought and salinity stresses. - Plant Cell Environ. 33: 453-467, 2010.

Murata N., Allakhverdiev S.I., Nishiyama Y.: The mechanism of photoinhibition in vivo: Re-evaluation of the roles of catalase, $\alpha$-tocopherol, non-photochemical quenching, and electron transport. - BBA-Bioenergetics 1817: 1127-1133, 2012.

Nakatsuka T., Nishihara M., Mishiba K., Yamamura S.: Temporal expression of flavonoid biosynthesis: Related genes regulates flower pigmentation in gentian plants. - Plant Sci. 168: 13091318, 2005b.

Nakatsuka T., Nishihara M., Mishiba K., Yamamura S.: Two different mutations are involved in the formation of whiteflowered gentian plants. - Plant Sci. 169: 949-958, $2005 \mathrm{a}$.

Neill S.O., Gould K.S.: Anthocyanins in leaves: light attenuators or antioxidants? - Funct. Plant Biol. 30: 865-873, 2003.

Neill S.O., Gould K.S., Kilmartin P.A.: Antioxidant activities of red versus green leaves in Elatostema rugosum. - Plant Cell Environ. 25: 539-547, 2002.

Oancea S., Oprean L.: Anthocyanins, from biosynthesis in plants to human health benefits. - Acta Univ. Cib. E XV: 3-16, 2011.

Reddy V.S., Dash S., Reddy A.R.: Anthocyanin pathway in rice (Oryza sativa L): identification of a mutant showing dominant inhibition of anthocyanins in leaf and accumulation of proanthocyanidins in pericarp. - Theor. Appl. Genet. 91: 301-312, 1995.

Rice-Evans C., Miller N., Paganga G.: Antioxidant properties of phenolic compounds. - Trends Plant Sci. 2: 152-159, 1997.

Saha M.R., Hasan S.M.R., Akter R. et al.: In vitro free radical scavenging activity of methanol extract of the leaves of Mimusops elengi Linn. - Bangl. J. Vet. Med. 6: 197-202, 2008.

Schubert H., Andersson M., Snoeijs P.: Relationship between photosynthesis and non-photochemical quenching of chlorophyll fluorescence in two red algae with different carotenoid compositions. - Mar. Biol. 149: 1003-1013, 2006.

Szymańska R., Ślesak I., Orzechowska A., Kruk J.: Physiological and biochemical responses to high light and temperature stress in plants. - Environ. Exp. Bot. 139: 165-177, 2017.

Takahashi S., Murata N.: How do environmental stresses accelerate photoinhibition? - Trends Plant Sci. 13: 178-182, 2008.

Tanaka Y., Sasaki N., Ohmiya A.: Biosynthesis of plant pigments: anthocyanins, betalains and carotenoids. - Plant J. 54: 733 $749,2008$.

Tucić B., Vuleta A., Jovanović S.M.: Protective function of foliar anthocyanins: in situ experiments on a sun-exposed population of Iris pumila L. (Iridaceae). - Pol. J. Ecol. 57: 779-783, 2009.

Wang H., Cao G., Prior R.L.: Oxygen radical absorbing capacity 
of anthocyanins. - J. Agr. Food Chem. 45: 304-309, 1997.

Welford R.W.D., Clifton I.J., Turnbull J.J. et al:: Structural and mechanistic studies on anthocyanidin synthase catalysed oxidation of flavanone substrates the effect of C-2 stereochemistry on product selectivity and mechanism. - Org. Biomol. Chem. 3: 3117-3126, 2005.

Young A.J.: The photoprotective role of carotenoids in higher plants. - Physiol. Plantarum 83: 702-708, 1991.

$\mathrm{Yu}$ Z.C., Zhang Q.L., Zheng X.T. et al.: Anthocyanin accumulation in juvenile Schima superba leaves is a growth trade-off by consuming energy for adaptation to high light during summer. - J. Plant Ecol. 12: 507-518, 2019.

Yu Z.C., Zheng X.T., Lin W. et al.: Different photoprotection strategies for mid-and late-successional dominant tree species in a high-light environment in summer. - Environ. Exp. Bot. 171: 103927, 2020.

Zeng X.Q., Chow W.S., Su L.J. et al.: Protective effect of supplemental anthocyanins on Arabidopsis leaves under high light. - Physiol. Plantarum 138: 215-225, 2010.

Zhang K.M., Yu H.J., Shi K. et al.: Photoprotective roles of anthocyanins in Begonia semperflorens. - Plant Sci. 179: 202208, 2010.

Zhang T.J., Chow W.S., Liu X.T.: A magic red coat on the surface of young leaves: anthocyanins distributed in trichome layer protect Castanopsis fissa leaves from photoinhibition. - Tree Physiol. 36: 1296-1306, 2016.

Zhang T.J., Zheng J., Yu Z.C. et al.: Functional characteristics of phenolic compounds accumulated in young leaves of two subtropical forest tree species of different successional stages. - Tree Physiol. 38: 1486-1501, $2018 \mathrm{~b}$.

Zhang T.J., Zheng J., Yu Z.C. et al.: Variations in photoprotective potential along gradients of leaf development and plant succession in subtropical forests under contrasting irradiances. Environ. Exp. Bot. 154: 23-32, 2018 a.

Zhu H., Zhang T.J., Zhang P., Peng C.L.: Pigment patterns and photoprotection of anthocyanin in the young leaves of four dominant subtropical forest tree species in two successional stages under contrasting light conditions. - Tree Physiol. 36: 1092-1104, 2016.

(C) The authors. This is an open access article distributed under the terms of the Creative Commons BY-NC-ND Licence. 